\title{
Factores que influyen en la percepción del bienestar y flourishing de los docentes de lenguas en formación
}

\author{
Leopoldo Medina-Sánchez, Juan Ramón Guijarro-Ojeda y \\ Manuel Jesús Cardoso-Pulido \\ Universidad de Granada (España)
}

\begin{abstract}
Tras investigar sobre la literatura referente al bienestar y comprender los orígenes del crecimiento humano y las emociones de los docentes de lenguas en formación, hemos elaborado un estudio comparativo-causal con estudiantes del Grado en Educación Primaria de la Universidad de Granada. Los objetivos de nuestra investigación pretenden identificar las creencias que tiene este estudiantado acerca de su formación inicial en lenguas extranjeras en base a los siguientes factores (variables dependientes): inteligencia emocional; angustia docente; malestar docente; éxito en la vida escolar y; apoyos percibidos por familiares, amigos y la sociedad. Asimismo, nuestro propósito se centra en comprender si las variables atributivas (variables independientes) -tales como género, lugar de nacimiento, profesión de los padres, etc.- son estadísticamente significativas cuando se cruzan con las variables dependientes. Como resultado principal obtenemos que los estudiantes que deciden inscribirse en el grado con una motivación vocacional se sienten apoyados en sus entornos sociales. Sin embargo, aquellas y aquellos estudiantes con una motivación funcional no perciben apoyos por lo que es más probable que abandonen la profesión de forma temprana. Igualmente, las respuestas otorgadas por nuestra cohorte arrojan luz sobre las modificaciones que necesitan aplicar los sistemas de educación superior con el fin de mejorar la calidad de la praxis docente durante el periodo de prácticas externas, el cual va a moldear su rol como futuras y futuros docentes de lenguas.
\end{abstract}

Palabras clave: Bienestar docente, ILE, docentes en formación.

Factors shaping pre-service foreign language teachers' perceptions on wellbeing and human flourishing. After delving into the literature about wellbeing and comprehending the core of preservice teachers' emotions and human flourishing, we developed a casual-comparative study with students at the Faculty of Educational Sciences (University of Granada). Our research aims to identify the senior students' beliefs about their teacher training programme in Foreign Languages (English) with reference to the following factors (dependent variables): emotional intelligence; teacher distress; teacher burnout; educational success and; perceived support from family, friends and society. Additionally, our purpose is to unravel whether the attributes variables -such as gender, birthplace, parents' profession...-indicate statistically significant or not when crossed with the dependent variables. Significant results pointed out that students who decided to join the teaching career with a vocational orientation perceived total support from their close social network. On the contrary, those with a functional reason do not observe any assistance and hence, they are willing to leave the teaching profession sooner. Finally, based on our research findings, we might shed light on improving the teaching experience as well as the upcoming transformations that higher educational systems have to apply in order to meet the real formative needs of pre-service EFL teachers.

Keywords: Teacher wellbeing, EFL, pre-service teachers.

Correspondencia: Manuel Jesús Cardoso-Pulido. Facultad de Ciencias de la Educación. Universidad de Granada. Campus de Cartuja. C.P.: 18071. Granada (España).

E-mail: $\underline{\text { manuelcardosopulido@gmail.com }}$ 
Nuestra investigación se ubica dentro del ámbito del estudio del bienestar docente. Concretamente, nos centramos en el bienestar del profesorado de Primaria en formación. Como aseveran numerosos expertos internacionales en materia de educación, las responsabilidades y roles del profesorado deben cuidarse desde la formación inicial, con el fin de otorgar a los futuros educadores herramientas con las que aumentar su bienestar personal y profesional y evitar así que continúen los casos de malestar y burnout (Delors, 1996; Esteve, 2003; Pérez, 2006; Cardoso-Pulido, 2016; Cardoso-Pulido y Guijarro-Ojeda, 2017; Medina y Pérez, 2017). En palabras de Brighouse (2002), reconsiderar la formación inicial docente generará liderazgo y pasión, y para Hargreaves (1997) traerá consigo una revolución para (re)educar con “corazón y mente”.

En lo que respecta a la literatura sobre el bienestar de los docentes en formación debemos destacar que se han elaborado escasas investigaciones desde este prisma. En primer lugar, el concepto de bienestar docente no fue estudiado hasta finales del siglo XX y principios del XXI (Smylie, 1999; Hoy y Miskel, 1996; Vanderberghe y Huberman, 1999; Hallinger, 2003), puesto que con anterioridad las investigaciones se centraban en los casos de malestar y abandono. Finalmente, el concepto de bienestar docente se compone de varias dimensiones definidas en el multi-modelo de Horn et al. (2002): 1) bienestar afectivo: satisfacción en el lugar de trabajo; 2) bienestar profesional: autonomía y aspiraciones profesionales; 3) bienestar social: fomento de las relaciones positivas entre alumnado y profesorado, entusiasmo y pasión por la enseñanza; 4) bienestar cognitivo: la concentración cuando la/el docente se enfrenta a nuevas situaciones; 5) bienestar psicosomático: ausencia o presencia de afecciones psicosomáticas.

Entre los estudios realizados sobre bienestar docente y cómo aumentarlo en las escuelas destacamos el éxito de los "programas ecológicos” realizados en Reino Unido, los cuales se caracterizan por una excelente comunicación y relación entre los miembros de la escuela, toma de decisiones conjunta basada en los valores del profesorado, disposición de los instrumentos y medios adecuados para la docencia así como la asignación de responsabilidades basada en las afinidades y habilidades de los docentes (Cartwright y Cooper, 1997; Comité de Educación de Londres, 1998; Rogers, 1996, 2006).

De manera específica, nuestro estudio pretende arrojar luz sobre el bienestar de los docentes de lenguas en formación, ámbito escasamente investigado. Cabe destacar aquí el trabajo de Borg (2006), quien coincide con otros muchos investigadores, entre ellos Hammadou y Bernhard (1987), en afirmar que enseñar una lengua extranjera (a partir de ahora, LE) es algo único y que difiere del resto de asignaturas. Borg (2006) llevó a cabo un estudio de corte cualitativo, organizando grupos focales con futuros docentes de LE (2006). En sus narrativas, los participantes reconocen la especificidad del hecho de ser docente de LE ("language teachers teach communication, not facts", Borg, ibíd.: 5). Igualmente, estos informantes declararon que sus relaciones con el alumnado eran más 
positivas y cercanas dado que las actividades propias de la enseñanza de lenguas están enfocadas en las experiencias personales que ayudaban a construir espacios seguros y agradables para todos.

En lo que respecta a la motivación y conocimientos previos en torno a la profesión docente, los estudiantes de Magisterio -asevera Pajares (1992)- se matriculan en los estudios de Educación Primaria e Infantil con ideas preconcebidas sobre lo que es la enseñanza. Este hecho se debe a que un/a futuro/a docente ha sido alumno/a durante al menos 20 años por lo que ha observado y experimentado lo que es ser un buen docente, o no. Por lo tanto, tanto las percepciones negativas como las positivas sobre la docencia y las buenas prácticas están arraigadas en el estudiantado. No obstante, Marso y Pigge (1989) afirman que, de manera general, los futuros docentes se matriculan en los estudios con una motivación intrínseca positiva. Asimismo, varias investigaciones indican que aquellos estudiantes con malas experiencias como alumnos en el sistema educativo son lo que más se matriculan con el objetivo de cambiar los métodos que utilizaron con ellos (Bird et al., 1992; Gupta y Saravanan, 1995; Calderhed y Robson, 1991; Cardoso-Pulido y Guijarro-Ojeda, 2017). Asimismo, Richardson (1996) explica que hay tres áreas principales que moldean las motivaciones de los docentes en formación: vida personal, vida académica y experiencias con el conocimiento formal.

Como observamos, el campo de la investigación en materia del bienestar de los docentes de LE en formación necesita estudios que arrojen luz sobre cómo desarrollar de manera efectiva las dimensiones sociales, afectivas y motivacionales dentro de la profesión y formación docente, con el fin de elevar su bienestar y su éxito en la vida escolar. Asimismo, es necesario que los futuros docentes adquieran estas cualidades y herramientas de trabajo si esperamos que puedan transmitirlas a su alumnado puesto que no se puede enseñar una competencia que previamente no se ha aprendido (Palomera et al., 2008). Para ello, hemos elaborado un estudio comparativo-causal con estudiantes del Grado en Educación Primaria de la Universidad de Granada. Asimismo, el objetivo principal de nuestro estudio pretende identificar las creencias que tienen los futuros docentes de LE acerca de su formación inicial en base a las siguientes variables: angustia docente; inteligencia emocional; malestar docente; éxito en la vida escolar y; apoyos percibidos por familiares, amigos y la sociedad.

De la misma manera, nuestro propósito se centra en comprender si las variables atributivas (variables independientes) -tales como género, lugar de nacimiento, profesión de los padres, zona y lugar del centro y motivación para cursar los estudios- son estadísticamente significativas cuando se cruzan con las variables dependientes (anteriormente citadas). 


\section{MÉTODO}

\section{Participantes}

El número total de informantes de nuestro estudio es de 88 futuros docentes de lengua extranjera durante su último año de estudios. De estos, 58 informantes pertenecen al plan de estudios monolingüe y 30 al plan bilingüe del Grado en Educación Primaria (mención LE, inglés) de la Universidad de Granada. La media de edad es de 23 años. El número de hombres que participaron en este estudio asciende a 25 , mientras que el número de mujeres se eleva a 63 . Asimismo, más del $90 \%$ de nuestros informantes posee un expediente académico de notable (entre 7 y 8.99 ).

\section{Instrumentos}

Los instrumentos que hemos empleado para llevar a cabo nuestro estudio son escalas estandarizadas:

Preguntas sociodemográficas e interrogantes abiertos sobre sus motivaciones y sentimientos. Teacher Distress Questionnaire. Mide la variable angustia profesional del docente. Fue realizado por Nieves de Jesús y Conboy (2001). El cuestionario tiene un total de 40 ítems, y está conformado por cinco subescalas (motivación intrínseca; expectativas sobre el buen desempeño profesional; angustia profesional; agotamiento profesional y; creencias irracionales). Se trata de una escala Likert que va desde 1 (totalmente en desacuerdo) a 7 (totalmente de acuerdo).

Trait Emotional Questionnaire-Short Form. Valora la variable inteligencia emocional del docente. Realizado por Petrides y Furham (2006). El cuestionario posee un total de 30 ítems conformado por seis dimensiones (emocionalidad; autocontrol; bienestar; sociabilidad; automotivación y; adaptabilidad). El cuestionario presenta reverse scores. Se trata de una escala Likert que va desde 1 (totalmente en desacuerdo) a 7 (totalmente de acuerdo).

Maslach Burnout Inventory-Educators Survey. Calcula la variable síndrome del quemado en la profesión docente. Realizado por Maslach, Jackson, y Leiter (1996). El cuestionario se compone de 22 ítems que se recogen en tres dimensiones (agotamiento emocional; despersonalización y; realización personal). Es una escala Likert que va desde 0 (nunca) a 6 (todos los días) y también presenta reverse scores.

Adaptación de la Rueda de la Vida Escolar. Determina la variable éxito en la vida escolar. Realizada por López-Pérez y Valls-Ballesteros (2013). La escala mide 12 dimensiones globales (creatividad; fuerza de voluntad y constancia; capacidad de superación ante dificultades; capacidad para discrepar por ti mismo; emociones y asertividad; capacidad de atención y concentración; liderazgo; participación en clase; relación con los alumnos y; relación con los compañeros de trabajo). Se trata de una escala 
tipo Likert que va desde 1 (muy poca satisfacción y poco éxito) a 10 (bastante satisfacción y éxito).

\section{Procedimiento}

El muestreo que hemos realizado es un muestreo probabilístico por conglomerado ya que los informantes componen una conglomeración natural (Bisquerra, 1989). En relación con la muestra objeto de nuestro estudio hemos seleccionado aquellos estudiantes de cuarto curso que asistían a sus prácticas externas como docentes de LE. Podríamos afirmar que también se trata de un estudio etnográfico ya que todos los participantes son granadinos y/o andaluces. El contacto con la muestra se llevó a cabo en dos cursos académicos, el 13 de mayo de 2016 y el 26 de mayo de 2017. Consideramos oportuno repetir el proceso de investigación con el fin de obtener más informantes.

\section{Análisis de datos}

Para el análisis de la información cuantitativa recolectada hemos utilizado el programa de análisis de datos cuantitativos SPSS v.24 y la hoja de cálculo Excel. En aras de comprimir y facilitar a los lectores la información que se arroja de nuestro estudio expondremos los resultados más significativos de manera narrativa en lugar de usar tablas que puedan dar lugar a confusión.

Para determinar si existen o no diferencias estadísticamente significativas en los cruces de las variables de identificación con los ítems de la escala de recogida de información hemos implementado las adecuadas pruebas de significación de naturaleza no paramétrica ante la violación de alguno de los supuestos paramétricos pertinentes.

\section{RESULTADOS}

Los resultados obtenidos, de manera general, tras la implementación de nuestra metodología son: Los participantes poseen niveles altos en las dimensiones positivas de la profesión docente (motivación intrínseca y; expectativas sobre el buen desempeño profesional) así como niveles bajos en los negativos (angustia profesional; agotamiento profesional y; creencias irracionales).

Los futuros docentes de lenguas han señalado como aspectos más importantes e influyentes en su praxis las dimensiones de adaptabilidad y bienestar.

Los participantes no poseen el síndrome del quemado en la profesión docente debido a sus bajas puntuaciones.

Los informantes otorgan la máxima puntuación a las relaciones con sus compañeros y las relaciones con el alumnado como sus puntos más fuertes frente al liderazgo con la puntuación más baja. 
De manera específica, los resultados más significativos de nuestro estudio son: El tipo de motivación para cursar los estudios es directamente proporcional al modo en que los futuros docentes se sienten apoyados por su círculo cercano. Los participantes con una motivación vocacional perciben total apoyo por parte de sus familiares y amigos frente a la crítica y desamparo que perciben los participantes con una motivación funcional hacia la carrera y profesión docente $(p<.001)$.

Los resultados arrojan que los informantes que han realizado sus prácticas externas en centros públicos obtienen mayores niveles de motivación intrínseca, seguidos de los que estaban en centro concertados y privados, respectivamente $(p<.01)$.

Sorprendentemente, los participantes con padres docentes manifestaron menor capacidad de autocontrol de manera significativa $(p<.05)$.

Los futuros docentes de LE con una motivación vocacional obtuvieron puntuaciones estadísticamente significativas superiores en las dimensiones de sociabilidad y automotivación $(p<.05)$.

Los participantes con una motivación funcional alcanzaron niveles estadísticamente altos en la dimensión (no) realización personal $(p<.05)$ llegando a ser el colectivo que otorga mayores puntuaciones en la escala Maslach Burnout Inventory.

Los informantes con tradición docente -padres que también lo son- obtienen puntuaciones estadísticamente significativas en la dimensión emociones y asertividad $(p<.01)$.

\section{DISCUSIÓN Y CONCLUSIONES}

El análisis de los datos pone de relieve, de forma significativa, que las motivaciones del estudiantado moldean e influyen en la calidad de la praxis docente ya que los participantes con una motivación vocacional perciben más bienestar que el resto de la cohorte (Horn et al., 2002). Asimismo, se pone de manifiesto que el bienestar personal y profesional que perciben viene influenciado por la calidad de sus relaciones sociales (Hargreaves, 1997; Rogers, 1999). De la misma manera, los participantes con una motivación vocacional tienden a socializar más, experimentado subidas en sus niveles de bienestar (Cardoso-Pulido y Guijarro-Ojeda, 2017; Cardoso-Pulido y Guijarro-Ojeda, 2017; Horn et al., 2002).

Los resultados principales muestran que los futuros docentes de lenguas con una orientación vocacional hacia la carrera y profesión docente poseen mayores niveles de bienestar y se sienten más apreciados por sus círculos sociales cercanos, por lo que será más probable desarrollar en ellos durante la formación inicial nuevos e innovadores métodos didácticos enfocados en las técnicas del bienestar. Por el contrario, aquellos con una motivación instrumental y/o con una motivación funcional poseen bajos niveles de 
compromiso hacia la profesión y formación docente y no perciben apoyos por parte de su entorno cercano inclinándose a abandonar la profesión de manera temprana.

Hemos observado que casi la totalidad de los informantes se ha inscrito en la carrera docente con una predisposición positiva (Marso y Pigge, 1989). Asimismo, los resultados ponen de manifiesto que para obtener éxito en la vida profesional del docente la formación inicial debe incrementar contenido en bienestar, emocionalidad, sociabilidad, autocontrol, motivación y adaptabilidad, siendo la adaptabilidad la cualidad que mayor incidencia tiene en el bienestar afectivo y positividad en el aula (Goleman, 1995; Palomera et al., 2008; Pérez-Valverde y Ruiz-Cecilia, 2014; Bird et al., 1992; Richardson, 1996; Calderhed y Robson, 1991; el comité de Educación de Londres, 1998).

Finalmente, hemos tratado de arrojar luz sobre las dinámicas que generan o apoyan el bienestar de los docentes de lenguas con el fin de proporcionar un marco teórico y unos resultados que desemboquen en la creación de recursos prácticos a través de enfoques humanísticos, sobre todo para las y los docentes que tienen problemas dentro del contexto escolar (malestar, estrés, rutina, trámites burocráticos...) o para las y los que desean emplear enfoques más humanísticos. En este sentido, los sistemas de educación superior deberían considerar la opción de rediseñar la formación inicial del profesorado de lenguas para que estos no solo sean simples vasijas transmisoras de un conocimiento formal sino también formadores de cultura e interculturalidad, así como animar al alumnado y al profesorado a cruzar las fronteras de las distintas disciplinas con el fin de reducir los estereotipos foráneos y comenzar a percibir otras culturas desde un punto de vista más exacto, es decir, superar las barreras del prejuicio hacia otras naciones reduciendo las diferencias entre las personas, independientemente de su lengua, cultura y/o ámbito social. Nosotros, como docentes de lenguas, tenemos que hacer conscientes a las alumnas y a los alumnos de que la enseñanza y el aprendizaje de una lengua extranjera no supone aprender cosas completamente nuevas dado que ya las conocemos en nuestra lengua materna. Sin embargo, sí implica aprender a comunicarse de una manera distinta y con personas distintas. Entonces, proponemos un aprendizaje de segundas y terceras lenguas como una competencia clave para el desarrollo profesional, personal y cultural. Tal y como hemos mencionado a lo largo de nuestro texto, el enfoque basado en el bienestar y en el crecimiento personal producirá cambios positivos en las mentalidades de nuestro alumnado siempre y cuando estos tengan un buen líder, es indudable que los cambios deben comenzar desde los niveles más elementales. Sin embargo, para que estas ideas y contenidos basados en el bienestar y el crecimiento personal puedan florecer es necesario que sean adquiridos primero durante la formación inicial del profesorado. 


\section{REFERENCIAS}

Bird, T., Anderson, L., Sullivan, B., y Swidler, S. (1992). Pedagogical balancing acts: A teacher educator encounters problems in an attempt to influence prospective teachers' beliefs. Washington, DC: Office of Educational Research and Improvement.

Bisquerra, R. (1989). Métodos de Investigación Educativa, Guía Práctica. Barcelona: Ediciones Ceac.

Borg, S. (2006). The distinctive characteristics of foreign language teachers. Language Teaching Research, 10(1), 3-31.

Brighouse, T. (2002). Passionate leadership. Nottingham: National College for School Leadership.

Calderhead, J., y Robson, M. (1991). Images of teaching: Student teachers' early conceptions of classroom practice. Teaching and Teacher Education, 7, 1-8.

Cardoso-Pulido, M.J., y Guijarro-Ojeda, J.R. (2017). Percepciones sobre el bienestar docente del profesorado de EFL en formación. Porta Linguarum, Monográfico II 2017, 157-172.

Cardoso-Pulido, M.J. (2016). Fundamentos teórico-prácticos del bienestar docente del profesorado de lenguas extranjeras. En Cristina Pérez Valverde (Ed.), La formación de docentes de lenguas extranjeras: hacia un desarrollo profesional profundo basado en la narratividad (pp. 93-108). Madrid: Visor Libros,.

Cartwright, S., y Cooper, C.L. (1997). Managing Workplace Stress. Londres: Sage.

Delors, J. (1996). Learning: The treasure within. Paris: International Commission on Education for the Twenty First Century, UNESCO.

Education Service Advisory Committee (1998). Managing Work-related Stress: a guide for managers and teachers in the schools. Londres: HMSO.

Esteve, J.M. (2003). La tercera revolución educativa. La educación en la sociedad del conocimiento. Barcelona: Paidós.

Goleman, D. (1995). Emotional Intelligence. New York: Bantam.

Gupta, R., y Saravanan, V. (1995). Old beliefs impede student teacher learning of reading instruction. Journal of Education for Teaching, 21, 347-360.

Hallinger, P. (2003). Leading educational change: re ections on the practice of instructional and transformational leadership. Cambridge Journal of Education, 33, 329-351.

Hammadou, J., y Bernhardt, E. (1987). On being and becoming a foreign language teacher. Theory into Practice, 26, 301-306.

Hargreaves, A. (1997). Rethinking educational change with heart and mind. Alejandria: The Association for Supervision and Curriculum Development Yearbook.

Horn, J.E., van, Taris, T.W., Schaufeli, W.B., y Shreurs, P.J.C. (2002). A multi- dimensional approach to measuring teacher well-being. Capítulo 3. Presentado para publicar, 64-81.

Hoy, W.K., y Miskel, C.G. (1996). Educational administration: Theory, research and practice. Nueva York: McGraw-Hill.

Jesus, S.N., y Conboy, J. (2001). A stress management course to prevent teacher distress. The International Journal of Educational Management, 15(3), 131-137.

López, C., y Valls, C. (2013). Coaching educativo. Las emociones, al servicio del aprendizaje. Madrid: SM.

Marso, R., y Pigge, F. (1989). The influence of preservice training and teaching experience upon attitude and concerns about teaching. Teaching and Teacher Education, 5, 33-41.

Maslach, C., Jackson, S.E., y Leiter, M.P. (1996). Maslach Burnout Inventory Manual, Third Edition. Palo Alto, CA: Consulting Psychologists Press. 
Medina, L., y Pérez, C. (2017). Adaptaciones Metodológicas en el Aula de Lengua Extranjera para el Alumnado con Discapacidad Intelectual: Estudio de Caso. Porta Linguarum, Monográfico II 2017, 267-282.

Pajares, F. (1992). Teachers' beliefs and educational research: Cleaning up a messy construct. Review of Educational Research, 62, 307-332.

Palomera, R., Fernández-Berrocal, P., y Brackett, M. (2008). La inteligencia emocional como una competencia básica en la formación inicial de los docentes: algunas evidencias. Electronic Journal of Research in Educational Psychology.

Pérez, C. (2006). The student teacher and the teaching role: searching for self-awareness models. International Journal of Learning, 12(9), 367-374.

Pérez, C., y Ruiz-Cecilia, R. (2014). The development of FL teachers' professional identity through the production of narratives. Porta Linguarum, 22, 61-72.

Petrides, K.V., y Furnham, A. (2001). Trait emotional intelligence: Psychometric investigation with reference to established trat taxonomies. European Journal of Personality, 15, 425-448.

Richardson, V. (1996). The role of attitudes and beliefs in learning to teach. En J. Siluka (Ed.), Handbook of research on teacher education, (2nd. ed, pp: 102-119). Nueva York: Macmillan.

Rogers, B. (1999). Towards a model for colleague support: Matching support to needs and contexts. Unpublished doctoral dissertation, Melbourne University.

Rogers, W.A. (1996). Managing Teacher Stress. Londres: Pitman.

Rogers, W.A. (2006). I get by with a little help... Colleague support in schools. Londres: Paul Chapman Publishing.

Smylie, M.A. (1999). Teacher stress in a time of reform. En R. Vandenberghe y A.M. Huberman (Eds.), Understanding and preventing teacher burnout (pp. 59-84). Cambridge: Cambridge University Press.

Vandenberghe, R., y Huberman, A.M (1999). Understanding and Preventing Teacher Burnout: A Source of International Research and Practice. Cambridge: Cambridge University Press.

Recibido: 15 de abril de 2019

Recepción Modificaciones: 29 de abril de 2019

Aceptado: 5 de mayo de 2019 